how to access additional expertise and support. The management team adapted national guidelines to take into account the resources available at the Nightingale. These guidelines were made available on the hospital intranet, and in a physical resource folder on the ward.

These interventions modestly improved confidence in palliative care from $65 \%$ to $68 \%$ over 3 months, but significantly improved for those with no previous experience, from $38 \%$ to $51 \%$. All training attendees reported feeling more confident. Those who received the most training saw confidence improve by $8 \%$, compared to $2 \%$ for those who received the least.

Working as a junior doctor leader ensured the palliative care management team were aware of and responded to day to day issues on the ward affecting patient care, helping make interventions to improve the service.

\section{Leading innovation and improvement}

\section{ESTABLISHING A STANDALONE MENTORSHIP SCHEME FOR NEWLY GRADUATED FYIIS DURING THE COVID-19 PANDEMIC: FEEDBACK AND EXPERIENCES}

Adam Pailing*, Huma Naqvi, Harriet Marsland. Sandwell and West Birmingham Hospitals NHS Trust, UK

\subsection{6/leader-2020-FMLM.55}

Aim A cross-site trust in Birmingham was tasked with setting up and providing a 6-week internship scheme for 24 newly graduated doctors from medical school as extra support in the midst of the COVID-19 pandemic, termed FYi1s.

Method A bespoke 1:1 buddy scheme between an FYi1 and current FY1 was opted for. Wherever possible, FYi1s were placed on the specialty and site they were starting on in August, and their buddy would be an FY1 currently working on that specialty. Feedback was obtained at the end of the internship. 16 of the 24 FYi1s responded to the feedback with a mixture of multiple choice and free text responses addressing communication, support, responsibility and the 1:1 buddy scheme.

Results Feedback from the internship was overwhelmingly positive and used to modify features of the full induction process in August, such as dedicated coffee meetings solely for new starters to share their experiences without senior presence.

The buddy system received excellent feedback and gave each FYi1 a personal support mechanism during their first weeks at the trust. Overcrowding on surgical wards was raised as an issue with a proportionally larger number of FYi1s starting on surgical rather than medical specialties volunteering to for the internship. Individual FYi1s would sometimes have to shadow different senior doctors each day if their buddy was taking annual leave or was working out-ofhours (eg. night shifts). Some FYi1s also did not manage to spend time on their August ward or specialty as that area remained a COVID zone throughout the whole of the internship.

Conclusions A comprehensive and successful mentorship scheme gives new employees invaluable experience and support to enable them to perform without limitation when they do begin work on their own in August.

\section{THE VALUE OF WRITTEN INFORMATION IN IMPROVING PATIENT EXPERIENCE}

T Crotty*, E Keane, T Moran, TP O'Dwyer, M Rafferty, SG Khoo. St. Vincents University Hospital, Nutley Lane, Dublin 4, IRE

\subsection{6/leader-2020-FMLM.56}

Flexible nasendoscopy (FNE) is a common procedure performed in ENT outpatient clinics. Although the majority of patients tolerate the procedure reasonably well, approximately $25 \%$ patients find the procedure distressing. Considering written information is cheap and widely accessible, we provided patients with an information leaflet prior to performing FNE and measured their satisfaction using a structured questionnaire.

Our results demonstrated a large improvement in patient satisfaction and understanding post-procedure. Additionally, patients found physicians who administered the information leaflet as having explained the procedure more comprehensively than without a questionnaire.

As healthcare professionals, we are committed to continually trying to improve patient experience. Providing leadership and innovation in healthcare can seem like a daunting task. However, small, simple changes can often result in large improvements in patient outcome. As leaders, we must increase awareness of the potential implications of small changes, such as written information on patient satisfaction.

\section{Quality Improvement, Leadership, Workforce Wellbeing}

\section{FATIGUE \& FACILITIES AT KETTERING GENERAL HOSPITAL THE IMPORTANCE OF HIGH-QUALITY REST TO MAXIMISE THE PERFORMANCE OF JUNIOR DOCTORS AND ENSURE PATIENT SAFETY}

Geeth Silva, Aiken Yam, Jessica Court, Rabia Imtiaz.

\subsection{6/leader-2020-FMLM.57}

Introduction Junior doctors are increasingly working in an overstretched NHS. In 2018 Kettering General Hospital (KGH) was awarded $£ 60,800$ of government funds to create high-quality rest facilities and improve junior doctor wellbeing.

Methods Through auditing doctors working at $\mathrm{KGH}$, alongside consulting senior management, a project initiation document was constructed to spend this money. From November 2019 to June $2020 £ 46,275$ was spent on creating new rest facilities, including separate sleeping and working areas. Furthermore, the purchase of modern furniture and equipment met the functional needs of busy junior doctors. One month after the completion of the project, the team conducted a post-action review by reauditing how the changes impacted morale, wellbeing and quality of patient care.

Results Now, the majority of doctors are happy with the current rest areas on offer at $\mathrm{KGH}(60 \%)$, and a majority feel that they will use the on-call room area (63\%). Overall, the feedback from both interviews and the JDF was positive and, the renovation improved morale and wellbeing. There was an 
increased ability to take breaks. However, the majority of doctors are still not exception-reporting missing breaks: 79\% (2019), 74\% (2020).

Lessons Learnt Whilst improved rest facilities were welcomed, this report recommends the maintenance of increased staffing levels and fatigue facilities during the recovery phase of COVID-19. The remaining $£ 14,524.38$ should be directed at creating shower facilities, upgrading computer hardware and sustaining the quality of $\mathrm{KGH}$ fatigue facilities. Lastly, the rate of exception-reporting must be increased through improving awareness, exploring alternative methods and supporting the action when necessary. The continual investment into rest facilities not only ensure workforce wellbeing but undoubtedly translates into the safety of our patients.

\section{Geriatrics, community resources, student leadership, patient-driven research}

\section{AN INNOVATIVE PATIENT DRIVEN PROTOCOL FOR THE DEVELOPMENT OF A CRISIS PREPAREDNESS TOOLKIT: CMU-CARES [CRISIS AVOIDANCE FOR RURAL ELDERLY STAKEHOLDERS]}

Brenda Varriano, Averie Dickinson*, Raha Mouzoon, Jacob Long, Ruoxi Lu, Andrea Beatty, Neli Ragina, Jyotsna Pandey. Central Michigan University (CMU) College of Medicine, Mt. Pleasant, Michigan, USA

\subsection{6/leader-2020-FMLM.58}

The COVID-19 pandemic challenged society, requiring measures that impacted communities socially and economically. Rurally dispersed older adults [RDOA] have a greater number of health comorbidities, poorer finances and limited access to internet and community resources (e.g. healthcare specialists), leaving them more vulnerable in a crisis. Current guidelines are better suited to urban communities, neglect certain needs of RDOA, and are at times difficult to navigate/access due to their online formats. RDOA specific resources are warranted and development should involve stakeholders. CMU-CARES sought to develop an innovative protocol that would engage RDOA in the development of a crisis preparedness toolkit. CMU-CARES protocol consists of six stages. (1) Awareness by communitybased outreach (e.g. mailed-letters, social media, community organizations) and participation from older adults in previous studies with the college of medicine. (2) Engagement occurred once a participant expressed interest. (3) Screening was completed at time of engagement. (4) Interviews/Interactions were modified from the WHOQOL-BREF and completed via WebEx online software to observe social distancing requirements. (5) Transcript analysis was completed with NVivo software to identify participant identified themes. (6) Design and development of a toolkit based on interviews. A target sample limited to 20-25 RDOA was used to reach data saturation. Presently 20 interviews have been completed. Preliminary themes include the need for timely and reliable information, training in use of technology and the need for social interaction. Overall, CMUCARES is a novel person-centered project dedicated to improving well-being and healthcare outcomes in the vulnerable RDOA population during crises. It hopes to foster camaraderie between healthcare providers and the community while inspiring medical student driven research and fostering careers in geriatrics, primary care and public health policy.
Leadership lessons from across the world

\section{TURNING ADVERSITY INTO OPPORTUNITY: UNDERGRADUATE LED IMPROVISATION IN TEACHING METHODS}

Anvita Anne, Varun Kumar Bandi, Satyanarayana Murthy Pusuluri. Dr. Pinnamaneni Siddhartha Institute of Medical Sciences and RF, Gannavaram, Andhra Pradesh, India

\subsection{6/leader-2020-FMLM.59}

Introduction There is a need for simple teaching methods every teacher can adopt, which are accepted by the students and provide a sustainable outcome. The traditional teaching methods do not achieve the desired outcomes, with many students left wanting for more practice of procedural skills.

Methodology MBBS Phase I students were randomly distributed into 3 groups: Group 1 (traditional method, $n=30$ ), Group 2 (Peyton's method, $\mathrm{n}=30$ ) and Group 3 (Modified Peyton's method, $\mathrm{n}=30$ ), and study duration was 6 months. Students were taught anatomy of the neck veins and were taught central venous catheterizations (CVC) into the right internal jugular vein by a single nephrologist on a mannequin. The modified Peyton's method consists of: Demonstration and Deconstruction, Comprehension, Performance and Observation, Teacher and Peer Feedback. The students were followed up with 4-week and 12-week recall.

Results The mean percentages obtained at end of 4 weeks in Groups 1, 2 and 3 were $82 \pm 10 \%, 86.67 \pm 7 \%$, and 87.33 $\pm 6.9 \%$ respectively. The percentages obtained at end of 12 weeks were $74.5 \pm 7.6 \%, 80.5 \pm 7.5 \%$ and $80.3 \pm 7.1 \%$ respectively. There was a significant decrease in percentages in all the groups $(\mathrm{p}<0.001)$.

At 4 weeks, there was significant difference between Groups 1 and 2, Groups 1 and $3(\mathrm{p}<0.05)$, while difference between Group 2 and 3 was not significant. At 12 weeks, there was significant difference between Groups 1 and 2 $(\mathrm{p}<0.01)$, Groups 2 and $3(\mathrm{p}<0.01)$ while there was no significant difference between Groups 2 and 3. The number of students confident of performing the procedure in Groups 1, 2 and 3 were 30\%, 56.7\% and 60\% respectively. There was significant difference in the confidence level between Group 1 and $2(\mathrm{p}<0.05)$, Group 1 and $3(\mathrm{p}<0.05)$, while the difference was non-significant between Group 2 and $3(\mathrm{p}=0.793)$.

Conclusion This study highlights the effort of a student in turning her adversity into an opportunity to improve the educational methods.

\section{Leading across systems and organisations}

\begin{tabular}{ll}
\hline 60 & A JOINT EFFORT AGAINST COVID-19: LEADING A \\
REMOTE REGIONAL TRAINING PROGRAMME AND \\
COLLABORATING WITH THE BRITISH SOCIETY FOR \\
RHEUMATOLOGY (BSR) ON A NATIONAL LEVEL
\end{tabular}

${ }^{1}$ Yik Long Man, ${ }^{2}$ Dalia Ludwig, ${ }^{2}$ James Glanville, ${ }^{3}$ Louise C Pollard, ${ }^{4}$ Claire Gorman, ${ }^{5}$ Jaita Mukherjee, ${ }^{6}$ Victoria Logan, ${ }^{6}$ Gerald Coakley, ${ }^{1}$ James Galloway. ${ }^{1}$ King's College Hospital NHS Foundation Trust, UK; ${ }^{2}$ University College London Hospital NHS Foundation Trust, UK; ${ }^{3}$ Lewisham and Greenwich NHS Trust, UK; ${ }^{4}$ Homerton University Hospital NHS Foundation Trust, UK; ${ }^{5}$ London North West University Healthcare NHS Trust, UK; ${ }^{6}$ Education and Trainee Committee, British Society for Rheumatology, UK

10.1136/leader-2020-FMLM.60 